\title{
Características quantitativas da carcaça de cordeiros terminados em confinamento e abatidos em diferentes condições corporais ${ }^{1}$
}

\section{Felipe Queiroga Cartaxo ${ }^{2}$, Marcílio Fontes Cezar ${ }^{3}$, Wandrick Hauss de Sousa ${ }^{4}$, Severino Gonzaga Neto ${ }^{5}$, José Morais Pereira Filho ${ }^{3}$, Maria das Graças Gomes Cunha ${ }^{4}$}

\footnotetext{
${ }^{1}$ Projeto financiado pelo convênio FINEP/EMEPA - PB.

2 Programa de Doutorado Integrado em Zootecnia/UFPB.

${ }^{3}$ Departamento de Medicina Veterinária/CSTR/UFCG - Patos, PB.

${ }^{4}$ EMEPA - PB

${ }^{5}$ Departamento de Zootecnia/CCA/UFPB - Areia, PB.
}

RESUMO - Objetivou-se avaliar os efeitos do grupo genético e da condição corporal sobre os rendimentos de carcaça de cordeiros terminados em confinamento. Foram utilizados 24 cordeiros Santa Inês e 24 cordeiros 1/2 Dorper $\times 1 / 2$ Santa Inês com peso médio de 19,8 kg e idade média de 103 dias. O período de adaptação às instalações e à dieta foi de 14 dias. Os animais receberam ração completa com 30\% de feno de maniçoba (Manihot pseudoglaziovii) e 70\% de concentrado. O delineamento experimental foi o inteiramente casualizado em esquema fatorial $2 \times 3$, composto de dois grupos genéticos e três condições corporais, com diferentes números de repetições. Não houve diferença entre os grupos genéticos para as características quantitativas estudadas, exceto o percentual de gordura interna, a espessura de gordura subcutânea na condição gorda e os rendimentos de perna e costela na condição corporal intermediária. O efeito da condição corporal dentro do grupo genético aumenta o percentual de gordura interna, a espessura de gordura subcutânea, o índice de compacidade da carcaça e os pesos dos cortes, exceto do pescoço, nos cordeiros Dorper $\times$ Santa Inês.

Palavras-chave: Dorper, escore corporal, gordura subcutânea, musculosidade, Santa Inês

\section{Quantitative traits of carcass from lambs finished in feedlot system and slaughtered at different body conditions}

\begin{abstract}
The objective of this study was to evaluate the effects of the genotypes and body condition on the carcass dressing of lambs finished in feedlot. Twenty-four Santa Inês and 24 1/2 Dorper $\times 1 / 2$ Santa Inês crossbred lambs averaging $19.8 \mathrm{~kg}$ of weight and 103 days old were used in the trial. The adaptation period to the facilities and diet was of 14 days. The animals received complete ration with $30 \%$ of maniçoba hay (Manihot pseudoglaziovii) and $70 \%$ of concentrate. The experimental design consisted of a completely randomized $2 \times 3$ factorial design (two genotypes and three corporal conditions) with different number of replicates. No differences between genotypes for the quantitative carcass traits studied were observed, except for the percentage of internal fat, fat thickness in the fat condition and dressing of the leg and rib in the intermediate corporal condition. The effect of the body condition within the genotype increases the internal fat percentage, fat thickness, compactness index of the carcass and weights of the saleable cuts, except for the weight of the neck in Dorper $\times$ Santa Inês crossbred lambs.
\end{abstract}

Key Words: body score, Dorper, muscularity, Santa Inês, subcutaneous fat

\section{Introdução}

O consumo de carne ovina no Brasil ainda é pouco expressivo, tanto em valores absolutos quanto em valores comparativos às demais carnes (Almeida Jr. et al., 2004). Segundo Oliveira et al. (2002), esse baixo consumo pode estar relacionado à falta de hábito, o que, todavia, pode ser contornado pela oferta, durante todo o ano, de produtos de qualidade e com oferta.
No processo de produção de carne ovina, a obtenção de carcaças com deposição adequada de gordura e carne mais tenra são aspectos interessantes para os consumidores, que exigem qualidade dos produtos (Frescura et al., 2005). De acordo com Bueno et al. (2000), a padronização das carcaças de cordeiros é necessária para valorizar o produto e atrair consumidores.

Essas características podem ser otimizadas com o uso de sistemas adequados de terminação e cruzamentos. A 
prática de terminação de ovinos em confinamento possibilitaria disponibilizar ao mercado consumidor animais mais jovem, com melhores características de carcaça, o que contribuiria para a expansão do consumo (Reis et al., 2001). Neste sentido, Bonagurio et al. (2003) afirmaram que o foco da produção mundial de carne mudou de quantidade para qualidade, uma vez que o produtor é obrigado a utilizar técnicas para melhorar sua produção, como o uso de cruzamentos.

Segundo Sousa et al. (2003), a raça Santa Inês é encontrada em todas as regiões do Brasil e consiste em boa opção para utilização em sistemas de cruzamento. De acordo com Sousa \& Leite (2000), a raça Dorper foi desenvolvida para atender a um único propósito, produção eficiente de carne, pois possui melhor qualidade de carcaça, com boa conformação e distribuição de gordura.

Estudos de Furusho-Garcia et al. (2000) comprovaram superioridade das características de carcaça dos animais mestiços em relação às de cordeiros Santa Inês puros, o que confirma a vantagem de utilização de raça especializada para corte. A determinação de acabamento da carcaça de cordeiros utilizando-se uma medida subjetiva, como a condição corporal, pode possibilitar o fornecimento aos consumidores de carne com adequada cobertura de gordura, de fácil aplicabilidade para os produtores.

Assim, realizou-se este trabalho para avaliar as características quantitativas de carcaças de cordeiros da raça Santa Inês e Dorper $\times$ Santa Inês terminados em confinamento e abatidos em diferentes condições corporais.

\section{Material e Métodos}

O experimento foi conduzido na Estação Experimental Pendência, base física da Empresa Estadual de Pesquisa Agropecuária da Paraíba (EMEPA-PB), localizada no município de Soledade, Paraíba. Foram utilizados 48 cordeiros não-castrados (24 da raça Santa Inês, todos Provi I ou II, e $24 \mathrm{~F}_{1}$ Dorper $\times$ Santa Inês) com idade média de 103 dias, pesando 20,4 e 19,2 kg, respectivamente.

$\mathrm{Na}$ fase de cria, os animais foram mantidos em regime semi-extensivo e, antes do alojamento, foram vacinados contra clostridiose e vermifugados com cloridrato de levamisol a 7,5\%, com uma repetição, após 15 dias, com vermífugo injetável (ivermectina a 1\%). Durante a gestação, as ovelhas mães dos cordeiros foram vacinadas contra clostridiose, motivo pelo qual foi feita apenas uma aplicação nesses animais.

Os animais foram distribuídos em baias individuais medindo $0,80 \times 1,20 \mathrm{~m}$, com livre acesso aos comedouros e bebedouros, onde foram mantidos em confinamento até atingirem a condição corporal preconizada para o abate. O período de adaptação foi de 14 dias e as pesagens dos cordeiros foram realizadas a cada 14 dias.

Utilizou-se dieta única, com relação volumoso: concentrado $30: 70$, com $16,0 \%$ de proteína bruta, 3,60\% de extrato etéreo, $28,0 \%$ de fibra em detergente neutro, $4,18 \%$ de matéria mineral e 2,70 Mcal de energia metabolizável por quilograma de matéria seca. Os níveis de proteína bruta ficaram abaixo dos $17 \%$ da matéria seca e os de energia metabolizável, abaixo dos 2,80 Mcal de energia metabolizável para cordeiros com $20 \mathrm{~kg}$ de peso vivo e ganho de peso médio diário de 300 g recomendadas pelo NRC (1985).

A dieta foi fornecida diariamente em duas refeições, às $7 \mathrm{~h} 30$ e $15 \mathrm{~h} 30$, para consumo de matéria seca correspondente a $5 \%$ do peso vivo, pesado e reajustado diariamente para manter nível de sobra de $10 \%$.

A avaliação do escore corporal foi feita por três examinadores, que classificaram os cordeiros em três condições corporais: magra (escores 1,0 e 2,0), intermediária (escores 2,5 e 3,5) e gorda (escores 4,0 e 5,0), de acordo com metodologia descrita por Osório \& Osório (2003). Para a atribuição dos escores, foram feitas avaliações semanais por meio de exame visual e palpação da região lombar dos cordeiros.

O critério para realização dos abates foi a condição corporal de cada grupo genético. Foi também estabelecido o tempo máximo de 56 dias de permanência dos cordeiros no confinamento. O primeiro abate foi realizado com os cordeiros na condição corporal magra com oito animais por grupo genético, o segundo com os cordeiros na condição corporal intermediária com oito animais por grupo genético e o terceiro abate quando os cordeiros atingiram a condição corporal gorda, aos 42 dias de confinamento. Dois cordeiros Santa Inês e um F1 Dorper × Santa Inês não atingiram a condição corporal gorda neste período e permaneceram no confinamento até 56 dias, período máximo preestabelecido para o fim da terminação, novamente não atingiram a condição corporal gorda e, por isso, foram classificados na condição corporal intermediária.

Os animais foram submetidos a jejum de 18 horas (sólido e líquido) e, antes do abate, foram pesados, para obtenção do peso vivo ao abate (PVA). Posteriormente, os animais foram suspensos pelas patas traseiras e insensibilizados por concussão cerebral. Em seguida, foram seccionadas as veias jugulares e as artérias carótidas, recolhendo-se o sangue em recipiente previamente tarado.

Após sangria e esfola, o conteúdo gastrintestinal foi retirado para determinação do peso corporal vazio (PCV), obtido da diferença entre o peso vivo ao abate (PVA) e o 
conteúdo gastrintestinal. As carcaças, sem pele, vísceras, cabeça, patas e órgãos genitais, foram pesadas para determinação do peso de carcaça quente (PCQ) e transportadas para câmara frigorífica a $4^{\circ} \mathrm{C}$, onde foram mantidas por 24 horas.

Depois de resfriadas, as carcaças foram pesadas para obtenção do peso da carcaça fria (PCF), calculando-se a porcentagem de perda por resfriamento (PR) pela fórmula $(\mathrm{PR} \%)=\mathrm{PCQ}-\mathrm{PCF} / \mathrm{PCQ} \times 100$. Em seguida, foram determinados os rendimentos de carcaça quente (RCQ) e de carcaça fria (RCF) e o rendimento biológico (RB), respectivamente, pelas seguintes fórmulas: $\mathrm{RCQ}=(\mathrm{PCQ} / \mathrm{PVA}) \times 100, \mathrm{RCF}=$ $(\mathrm{PCF} / \mathrm{PVA}) \times 100$ e RB $=(\mathrm{PCQ} / \mathrm{PCV}) \times 100$.

O cálculo do percentual de gordura interna foi obtido pelo somatório das gorduras renal, inguinal e pélvica em relação ao peso da carcaça fria. As gorduras mesentérica e omental, como não fazem parte da carcaça, foram eliminadas juntamente com vísceras.

Para obtenção dos cortes comerciais e determinação da composição regional, a carcaça foi seccionada ao meio com serra elétrica, marca G. Paniz, modelo SF 42, fabricada por Magno Equipamentos. Na meia-carcaça direita, mediu-se o comprimento interno da carcaça com auxílio de fita métrica, que foi dividido pelo peso da carcaça fria para determinação do índice de compacidade da carcaça (ICC).

Posteriormente, para determinação da composição regional, a meia-carcaça foi seccionada em cinco cortes comerciais, descritos a seguir:

- pescoço, separado da carcaça em sua extremidade inferior entre a última vértebra cervical e a primeira torácica;

- paleta, obtida pela secção da região axilar, por meio do corte dos tecidos que unem a escápula e o úmero à região torácica da carcaça;

- costelas, retiradas entre a última vértebra cervical e a primeira torácica, e a última vértebra torácica e a primeira lombar;

- lombo, obtido pelo corte da última vértebra torácica e a primeira lombar, e da última lombar e a primeira sacral;

- perna, separada da carcaça em sua extremidade superior entre a última lombar e a primeira sacral.

Todos os cortes da carcaça foram pesados para cálculo do rendimento dos cortes comerciais. Na meia-carcaça esquerda, realizou-se um corte transversal entre $12^{\underline{a}}$ e $13^{\underline{a}}$ costelas, expondo a secção transversal do músculo Longissimus lumborum, cuja área foi tracejada com caneta apropriada sobre uma película plástica transparente para determinação da área de olho-de-lombo (AOL). Foram obtidas, por meio de régua, a largura máxima (A) e a profundidade máxima (B) para determinação da área de olho-delombo, pela fórmula: $\mathrm{AOL}=(\mathrm{A} / 2 * \mathrm{~B} / 2) \pi$.
A espessura de gordura subcutânea (EGS) foi medida com paquímetro e obtida a três quartos de distância do lado medial do músculo Longissimus lumborum para seu lado lateral da linha dorso-lombar. As gorduras renal, pélvica e inguinal foram obtidas após o resfriamento e antes da obtenção da meia-carcaça.

O índice de musculosidade da perna dos cordeiros foi estimado pela dissecação dos tecidos da perna. A perna, depois de pesada, foi acondicionada em sacos plásticos e congelada em freezer $\left(-20^{\circ} \mathrm{C}\right)$. Posteriormente, realizou-se a separação dos tecidos muscular, ósseo e adiposo e mediu-se o fêmur para cálculo do índice de musculosidade da perna, segundo metodologia descrita por Purchas et al. (1991):

$$
I M P=\frac{\sqrt{P 5 M / C F}}{C F}
$$

em que: IMP = índice de musculosidade da perna; P5M = peso dos cinco músculos (bíceps femural, quadríceps femural, semimembranoso, semitendinoso e adutor); $\mathrm{CF}=$ comprimento do fêmur.

As variáveis quantitativas estudadas foram analisadas estatisticamente por análise de variância, em delineamento inteiramente casualizado em esquema fatorial $2 \times 3$ (dois grupos genéticos e três condições corporais) com número de repetições variando de acordo com a condição corporal, utilizando-se o teste F para comparação dos quadrados médios dos fatores testados e o teste Tukey a 5\% de probabilidade para comparação das médias.

O modelo estatístico utilizado foi:

$$
Y_{i j}=\mu+G_{i}+E_{i}+G_{i j}+\varepsilon_{i j} \text {, }
$$

em que: $\mathrm{Y}_{\mathrm{ij}}=$ valor observado da variável dependente estudada, $\mu=$ média geral; $G_{i}=$ efeito do grupo genético i; $E_{i}=$ efeito do escore $j ; \mathrm{GE}_{\mathrm{ij}}=$ interação grupo genético $\times$ escore e $\varepsilon_{\mathrm{ij}}=$ erro aleatório associado a cada observação.

\section{Resultados e Discussão}

Não houve interação significativa $(\mathrm{P}>0,05)$ grupo genético $\times$ condição corporal sobre as características quantitativas de carcaça, exceto o percentual de gordura interna (Tabela 1), portanto, os resultados serão apresentados de forma independente.

Os pesos de carcaça, os rendimentos e as perdas por resfriamento não diferiram $(\mathrm{P}>0,05)$ entre os grupos genéticos. O rendimento de carcaça quente nos cordeiros F1 Dorper $\times$ Santa Inês foi inferior aos 58,0\% encontrados por Notter et al. (2004). Provavelmente o maior peso vivo ao abate $(45,7 \mathrm{~kg})$ dos cordeiros influenciou esta diferença, 
Tabela 1 - Pesos e rendimentos de carcaça de cordeiros de dois grupos genéticos e três condições corporais

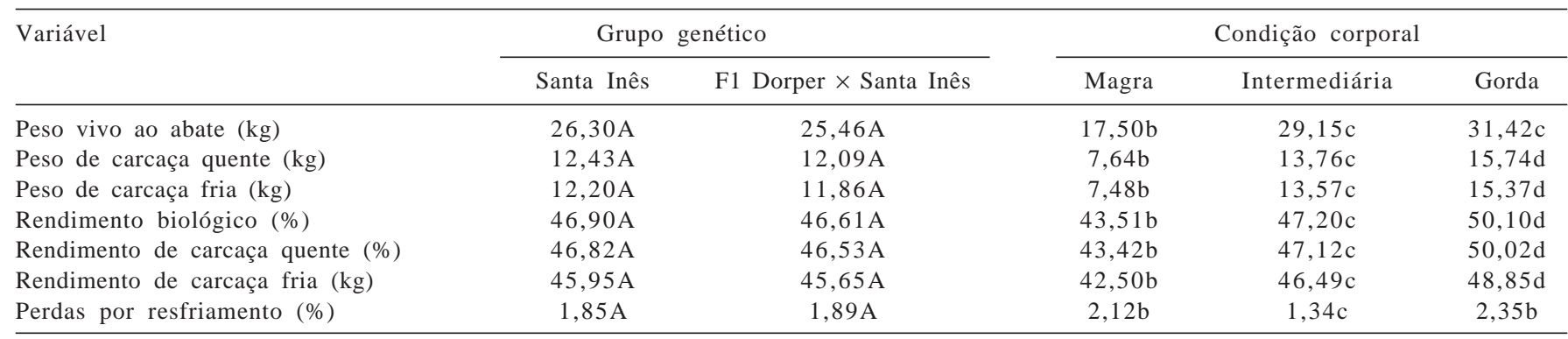

Letras distintas indicam diferenças significativas pelo teste Tukey a 5\% de probabilidade, maiúsculas para grupo genético e minúscula para condição corporal.

pois há correlação significativa e positiva entre peso vivo ao abate e rendimento de carcaça.

Da mesma forma, os rendimentos de carcaça quente e fria dos cordeiros Santa Inês com 46,82 e 45,95\%, respectivamente, foram inferiores aos 53,30 e 52,60\% encontrados por Oliveira et al. (2002) para ovinos Santa Inês e aos 53,10 e 53,10\% verificados para os cordeiros Bergamácia abatidos aos 43,70 kg. Entretanto, igualmente, esses autores também não observaram diferenças $(\mathrm{P}>0,05)$ nos rendimentos de carcaça entre os grupos genéticos.

Os resultados obtidos nesta pesquisa para os pesos e rendimentos de carcaça indicam que cordeiros Santa Inês com bom potencial genético, Provi I ou II, podem apresentar pesos e rendimentos de carcaça similares aos de seus cruzamentos. Embora os cordeiros F1 Dorper $\times$ Santa Inês tenham na sua composição genética $50 \%$ de uma raça especializada para corte, e considerando que a heterose na geração F1 é mais intensa, não foi observada superioridade dos cordeiros mestiços em relação aos Santa Inês.

As perdas por resfriamento das carcaças dos cordeiros entre grupos genéticos não foram significativas $(\mathrm{P}>0,05)$, o que confirma os resultados obtidos por Furusho-Garcia et al. (2000). Contudo, diferenças significativas para perdas por resfriamento entre grupos genéticos foram encontradas por Osório et al. (2002) ao avaliarem cordeiros Border Ideal e Border $\times$ Corriedale com 5,78 e 8,52\%, respectivamente.

A condição corporal influenciou $(\mathrm{P}<0,05)$ os pesos e rendimentos de carcaça, pois, com o aumento da condição corporal, os cordeiros apresentaram maiores pesos de carcaça quente e fria e, conseqüentemente, rendimentos de carcaça superiores. Da mesma forma, Peña et al. (2005) avaliaram carcaças de cordeiros Segureña com dois pesos de carcaça quente (8,1-10,0 e 10,1-13,0 kg) e encontraram diferença significativa $(\mathrm{P}<0,05)$ entre os rendimentos de carcaça quente e de carcaça fria.

Exceto na condição corporal intermediária, não houve efeito $(P>0,05)$ das condições corporais sobre as perdas por resfriamento. Esses resultados não eram previstos para a condição corporal intermediária em relação à gorda, pois a espessura de gordura subcutânea é o principal entrave para reduzir as perdas durante o resfriamento.

Houve influência significativa $(\mathrm{P}<0,05)$ do grupo genético e da condição corporal sobre o percentual de gordura interna (Tabela 2), assim, a deposição de gordura nestes grupos genéticos difere entre as condições corporais. Além disso, cordeiros Santa Inês apresentaram maior $(\mathrm{P}<0,05)$ percentual de gordura interna na condição corporal intermediária e os cordeiros F1 Dorper $\times$ Santa Inês apresentaram percentual mais elevado $(\mathrm{P}<0,05)$ na condição corporal gorda. Segundo Burke \& Apple (2007), há consenso nos trabalhos da literatura de que ovinos deslanados tendem a depositar maiores quantidades de gordura interna em comparação a raças lanadas. Os resultados encontrados neste estudo para condição corporal intermediária e gorda foram semelhantes aos obtidos por esses autores, que, ao avaliarem quatro grupos genéticos ovinos abatidos com 210 dias de idade terminados em pastejo e com suplementação com concentrado, verificaram que o percentual de gordura renal diferiu $(\mathrm{P}<0,05)$ entre grupos genéticos.

Como os cordeiros na condição corporal magra estavam na sua maioria com escore corporal 1,5 (muito magro), praticamente não havia gordura interna nestes animais, o que fez com que os desvios-padrão nesta condição ficassem altos.

As carcaças dos cordeiros Santa Inês e dos F1 Dorper $\times$ Santa Inês apresentaram semelhança para área de olhode-lombo, denotando que, na mesma condição corporal, a quantidade de tecido muscular similar é semelhante entre esses grupos genéticos, uma vez que esta a área é um indicativo de músculo na carcaça. Da mesma forma, Siqueira \& Fernandes (2000), estudando cordeiros puros Corriedale e mestiços Ile de France $\times$ Corriedale, não verificaram efeito do grupo genético sobre a área de olho-de-lombo. 
Tabela 2 - Porcentagem de gordura interna, área de olho-de-lombo, espessura de gordura subcutânea, índice de musculosidade da perna e índice de compacidade da carcaça em carcaças de cordeiros de dois grupos genéticos e três condições corporais

\begin{tabular}{|c|c|c|c|}
\hline Variável & \multicolumn{3}{|c|}{ Condição corporal } \\
\hline \multicolumn{4}{|l|}{ Gordura interna (\%) } \\
\hline $\begin{array}{l}\text { Santa Inês } \\
1 / 2 \text { Dorper } \times 1 / 2 \text { Santa Inês }\end{array}$ & $\begin{array}{l}0,10 \pm 0,09 \mathrm{aA} \\
0,32 \pm 0,44 \mathrm{aA}\end{array}$ & $\begin{array}{l}2,42 \pm 0,39 \mathrm{aB} \\
1,60 \pm 0,33 \mathrm{bB}\end{array}$ & $\begin{array}{l}2,29 \pm 0,43 \mathrm{aB} \\
2,84 \pm 0,90 \mathrm{bC}\end{array}$ \\
\hline \multicolumn{4}{|c|}{ Área de olho-de-lombo $\left(\mathrm{cm}^{2}\right)$} \\
\hline \multicolumn{4}{|c|}{ Espesssura de gordura subcutânea (mm) } \\
\hline $\begin{array}{l}\text { Santa Inês } \\
1 / 2 \text { Dorper } \times 1 / 2 \text { Santa Inês }\end{array}$ & $\begin{array}{l}0,23 \pm 0,42 \mathrm{aA} \\
0,25 \pm 0,46 \mathrm{aA}\end{array}$ & $\begin{array}{l}1,20 \pm 0,35 \mathrm{aB} \\
1,16 \pm 0,50 \mathrm{aB}\end{array}$ & $\begin{array}{l}1,25 \pm 0,40 \mathrm{aB} \\
2,00 \pm 0,50 \mathrm{bC}\end{array}$ \\
\hline \multicolumn{4}{|c|}{ Índice de musculosidade da perna } \\
\hline \multicolumn{4}{|c|}{ Índice de compacidade da carcaça $(\mathrm{kg} / \mathrm{cm})$} \\
\hline $\begin{array}{l}\text { Santa Inês } \\
1 / 2 \text { Dorper } \times 1 / 2 \text { Santa Inês }\end{array}$ & $\begin{array}{l}0,14 \pm 0,01 \mathrm{aA} \\
0,14 \pm 0,03 \mathrm{aA}\end{array}$ & $\begin{array}{l}0,23 \pm 0,02 \mathrm{aB} \\
0,22 \pm 0,02 \mathrm{aB}\end{array}$ & $\begin{array}{l}0,24 \pm 0,25 \mathrm{aB} \\
0,26 \pm 0,03 \mathrm{aC}\end{array}$ \\
\hline
\end{tabular}

Letras distintas (minúscula na coluna e maiúscula na linha) indicam diferenças significativas pelo teste Tukey a 5\% de probabilidade.

Condição corporal: magra (escores 1,0 a 2,0); intermediária (escores 2,5 a 3,5); gorda (escores 4,0 a 5,0).

A condição corporal gorda influenciou $(\mathrm{P}<0,05)$ a espessura de gordura subcutânea, que não diferiu nas demais condições corporais. Os cordeiros F1 Dorper $\times$ Santa Inês apresentaram maior espessura de gordura subcutânea e os Santa Inês, menor. Esse aumento da espessura de gordura subcutânea é importante para se obter melhor acabamento de carcaça, uma vez que adequada cobertura de gordura subcutânea é imprescindível para produção de melhores carcaças. Os resultados para condição gorda foram semelhantes aos obtidos por Oliveira et al. (2002), que, avaliando cordeiros Santa Inês e Bergamácia abatidos com peso de carcaça quente de 23,30 e 24,90 kg, respectivamente, verificaram menor $(\mathrm{P}>0,05)$ espessura de gordura de subcutânea nos cordeiros Santa Inês.

$\mathrm{O}$ efeito do grupo genético sobre os índices de musculosidade dos cordeiros foi significativo $(\mathrm{P}<0,05)$ apenas na condição corporal intermediária, na qual os cordeiros Santa Inês apresentaram 0,38 e foram inferiores aos F1 Dorper $\times$ Santa Inês $(0,43)$. Esse resultado se deve ao fato de os cordeiros F1 Dorper $\times$ Santa Inês terem apresentado menor $(\mathrm{P}<0,05)$ comprimento do fêmur. Silva Sobrinho et al. (2005), avaliando três grupos genéticos ovinos abatidos com peso de carcaça fria variando de 17,9 a 19,0 kg, portanto, superiores aos pesos deste estudo, encontraram diferenças significativas $(\mathrm{P}<0,05)$ entre grupos genéticos.

O efeito da condição corporal dentro de grupo genético sobre o percentual de gordura interna dos cordeiros
Santa Inês foi significativo $(\mathrm{P}<0,05)$ apenas na condição magra (Tabela 2), enquanto os F1 Dorper $\times$ Santa Inês foram diferentes $(\mathrm{P}<0,05)$ quanto ao acúmulo de gordura interna nas três condições corporais estudadas. Provavelmente a deposição mais rápida de gordura interna nos cordeiros Santa Inês seja uma característica da raça, enquanto, nos F1 Dorper $\times$ Santa Inês, por serem de raça especializada para corte, esta deposição aumente paulatinamente com estado de "engorduramento".

Da mesma forma, Frutos et al. (1995) avaliaram ovelhas adultas Churra e Merina abatidas com escore corporal menor que 2,5 e maior que 2,5 e encontraram diferenças $(\mathrm{P}<0,05)$ entre grupos genéticos para o total de gordura interna em relação ao peso de corpo vazio.

A área de olho-de-lombo na condição corporal magra diferiu $(\mathrm{P}<0,05)$ da observada nas condições intermediária e gorda, tanto nos cordeiros Santa Inês quanto nos F1 Dorper $\times$ Santa Inês. Isto comprova que, a partir da condição intermediária, praticamente cessou o crescimento de tecido muscular, independentemente do grupo genético.

Não houve efeito $(P>0,05)$ da condição corporal dentro do grupo genético sobre a espessura de gordura subcutânea nos cordeiros Santa Inês nas condições intermediária e gorda. Todavia, a espessura de gordura subcutânea encontrada nos cordeiros F1 Dorper $\times$ Santa Inês com condições corporais magra, intermediária e gorda foi diferente. Isto denota que os cordeiros F1 Dorper $\times$ Santa Inês apresentaram maior espessura de gordura subcutânea na condição 
corporal gorda em relação à intermediária, diferentemente dos cordeiros Santa Inês. Com base na área de olho-delombo e na espessura de gordura subcutânea, a condição corporal intermediária nos cordeiros F1 Dorper $\times$ Santa Inês pode ser preconizada para abate quando se deseja obter carcaça com proporção maior de músculo e menor de gordura subcutânea.

O índice de musculosidade da perna foi similar $(\mathrm{P}>0,05)$ entre cordeiros Santa Inês e F1 Dorper $\times$ Santa Inês nas três condições corporais estudadas. Houve influência $(\mathrm{P}<0,05)$ da condição corporal magra, em relação às demais, em ambos os grupos genéticos, o que indica que, após a condição corporal intermediária, praticamente não houve aumento de peso dos cinco músculos que compõem este índice, tampouco do crescimento do fêmur. Este fato também pode ser evidenciado pela área de olho-de-lombo, indicativo de tecido muscular na carcaça, que acompanhou o mesmo efeito.

O índice de compacidade da carcaça dos cordeiros Santa Inês na condição corporal magra diferiu $(\mathrm{P}<0,05)$ do obtido na condição corporal intermediária e a gorda, entretanto, os cordeiros F1 Dorper $\times$ Santa Inês apresentaram índices superiores $(\mathrm{P}<0,05)$ nas três condições corporais, o que pode ser justificado pelo maior peso de carcaça fria dos cordeiros F1 Dorper $\times$ Santa Inês nas diversas condições corporais, pois a outra variável que compõe este índice, o comprimento interno da carcaça, foi semelhante nas condições intermediária e gorda.
O índice de compacidade da carcaça não foi influenciado $(\mathrm{P}>0,05)$ pelos grupos genéticos. Da mesma forma, Siqueira \& Fernandes (2000), trabalhando com dois grupos genéticos, Corriedale e Corriedale $\times$ Ile de France, não encontraram diferenças para essa variável, o que possivelmente se deve à semelhança dos pesos de carcaça fria nas respectivas condições corporais entre os grupos genéticos.

Não houve influência $(\mathrm{P}>0,05)$ dos grupos genéticos sobre nenhum dos pesos dos cortes comerciais (Tabela 3). Esses resultados foram semelhantes aos obtidos por Oliveira et al. (2002), que, avaliando cordeiros Santa Inês e Bergamácia, não encontraram diferenças $(\mathrm{P}>0,05)$ nos cortes comerciais estudados entre grupos genéticos.

Os pesos dos cortes da carcaça dos cordeiros Santa Inês na condição corporal magra diferiram $(\mathrm{P}<0,05)$ dos obtidos nas condições intermediária e gorda. Entretanto, os cordeiros F1 Dorper $\times$ Santa Inês apresentaram diferenças $(\mathrm{P}<0,05)$ em todos os cortes nas três condições corporais, exceto o peso do pescoço, que não foi significativo $(\mathrm{P}>0,05)$ na condição corporal magra em relação às condições corporais intermediária e gorda.

Os cordeiros F1 Dorper $\times$ Santa Inês apresentaram diferenças $(\mathrm{P}<0,05)$ nos pesos de perna, lombo, paleta e costela em relação aos cordeiros Santa Inês nas condições corporais intermediária e gorda. Isso demonstra que os cordeiros F1 Dorper $\times$ Santa Inês atingiram, em comparação aos Santa Inês, maiores $(\mathrm{P}<0,05)$ pesos desses cortes quando atingiram a condição corporal gorda.

Tabela 3 - Pesos dos cortes comerciais de cordeiros de dois grupos genéticos e três condições corporais

\begin{tabular}{|c|c|c|c|}
\hline \multirow[t]{2}{*}{ Variável } & \multicolumn{3}{|c|}{ Condição corporal } \\
\hline & Magra & Intermediária & Gorda \\
\hline \multicolumn{4}{|l|}{ Peso da perna (kg) } \\
\hline Santa Inês & $1,29 \pm 0,13 \mathrm{aA}$ & $2,14 \pm 0,28 \mathrm{aB}$ & $2,25 \pm 0,32 \mathrm{aB}$ \\
\hline $1 / 2$ Dorper $\times 1 / 2$ Santa Inês & $1,22 \pm 0,35 \mathrm{aA}$ & $2,01 \pm 0,20 \mathrm{aB}$ & $2,48 \pm 0,31 \mathrm{aC}$ \\
\hline \multicolumn{4}{|l|}{ Peso do lombo (kg) } \\
\hline Santa Inês & $0,46 \pm 0,09 \mathrm{aA}$ & $0,92 \pm 0,18 \mathrm{aB}$ & $0,92 \pm 0,10 \mathrm{aB}$ \\
\hline $1 / 2$ Dorper $\times 1 / 2$ Santa Inês & $0,46 \pm 0,11 \mathrm{aA}$ & $0,86 \pm 0,14 \mathrm{aB}$ & $1,06 \pm 0,17 \mathrm{aC}$ \\
\hline \multicolumn{4}{|l|}{ Peso da paleta (kg) } \\
\hline Santa Inês & $0,80 \pm 0,07 \mathrm{aA}$ & $1,28 \pm 0,15 \mathrm{aB}$ & $1,37 \pm 0,17 \mathrm{aB}$ \\
\hline $1 / 2$ Dorper $\times 1 / 2$ Santa Inês & $0,76 \pm 0,21 \mathrm{aA}$ & $1,15 \pm 0,11 \mathrm{aB}$ & $1,35 \pm 0,20 \mathrm{aC}$ \\
\hline \multicolumn{4}{|l|}{ Peso do pescoço (kg) } \\
\hline Santa Inês & $0,33 \pm 0,04 \mathrm{aA}$ & $0,52 \pm 0,12 \mathrm{aB}$ & $0,55 \pm 0,15 \mathrm{aB}$ \\
\hline $1 / 2$ Dorper $\times 1 / 2$ Santa Inês & $0,31 \pm 0,07 \mathrm{aA}$ & $0,48 \pm 0,16 \mathrm{aB}$ & $0,55 \pm 0,11 \mathrm{aB}$ \\
\hline \multicolumn{4}{|l|}{ Peso da costela (kg) } \\
\hline Santa Inês & $0,90 \pm 0,14 \mathrm{aA}$ & $2,01 \pm 0,35 \mathrm{aB}$ & $1,95 \pm 0,34 \mathrm{aB}$ \\
\hline $1 / 2$ Dorper $\times 1 / 2$ Santa Inês & $0,91 \pm 0,23 a A$ & $1,55 \pm 0,49 \mathrm{aB}$ & $2,20 \pm 0,36 \mathrm{aC}$ \\
\hline
\end{tabular}

Letras distintas (minúscula na coluna e maiúscula na linha) indicam diferenças significativas pelo teste Tukey a 5\% de probabilidade.

Condição corporal: magra (escore corporal 1,0 a 2,0); intermediária (escore corporal 2,5 a 3,5); gorda (escore corporal 4,0 a 5,0). 
Não houve efeito $(\mathrm{P}>0,05)$ dos grupos genéticos sobre os percentuais dos demais cortes, exceto o da perna e das costelas na condição intermediária e o percentual da paleta na condição corporal gorda (Tabela 4). Os cordeiros F1 Dorper $\times$ Santa Inês foram superiores $(\mathrm{P}<0,05)$ aos Santa Inês quanto ao percentual da perna e inferiores $(\mathrm{P}<0,05)$ quanto ao percentual de costela. Na condição corporal intermediária, os cordeiros F1 Dorper $\times$ Santa Inês apresentaram vantagem em relação aos Santa Inês no rendimento da perna, pois é neste corte que se localiza o pernil, porção mais valorizada pelos consumidores.

Os cordeiros Santa Inês apresentaram maior $(\mathrm{P}<0,05)$ percentual de paleta na condição corporal gorda. Gutiérrez et al. (2005) avaliaram o percentual de paleta de três grupos genéticos com peso médio de carcaça fria de 15,4 kg e também verificaram diferenças significativas nos animais avaliados. Contudo, esses autores não observaram diferenças nos percentuais dos demais cortes.

Os rendimentos de perna e paleta diferiram $(\mathrm{P}<0,05)$ entre as condições corporais, enquanto os rendimentos de lombo e pescoço foram semelhantes $(\mathrm{P}>0,05)$ nas três condições corporais e em ambos os grupos genéticos. O percentual das costelas nos cordeiros Santa Inês foi significativo $(\mathrm{P}<0,05)$ nas condições corporais magra e intermediária, ambas semelhantes $(\mathrm{P}>0,05)$ à condição corporal gorda. Os cordeiros F1 Dorper $\times$ Santa Inês, no entanto, apresentaram similaridade $(\mathrm{P}>0,05)$ em todas as condições corporais.

Tabela 4 - Rendimentos dos cortes comerciais de cordeiros de dois grupos genéticos e três condições corporais

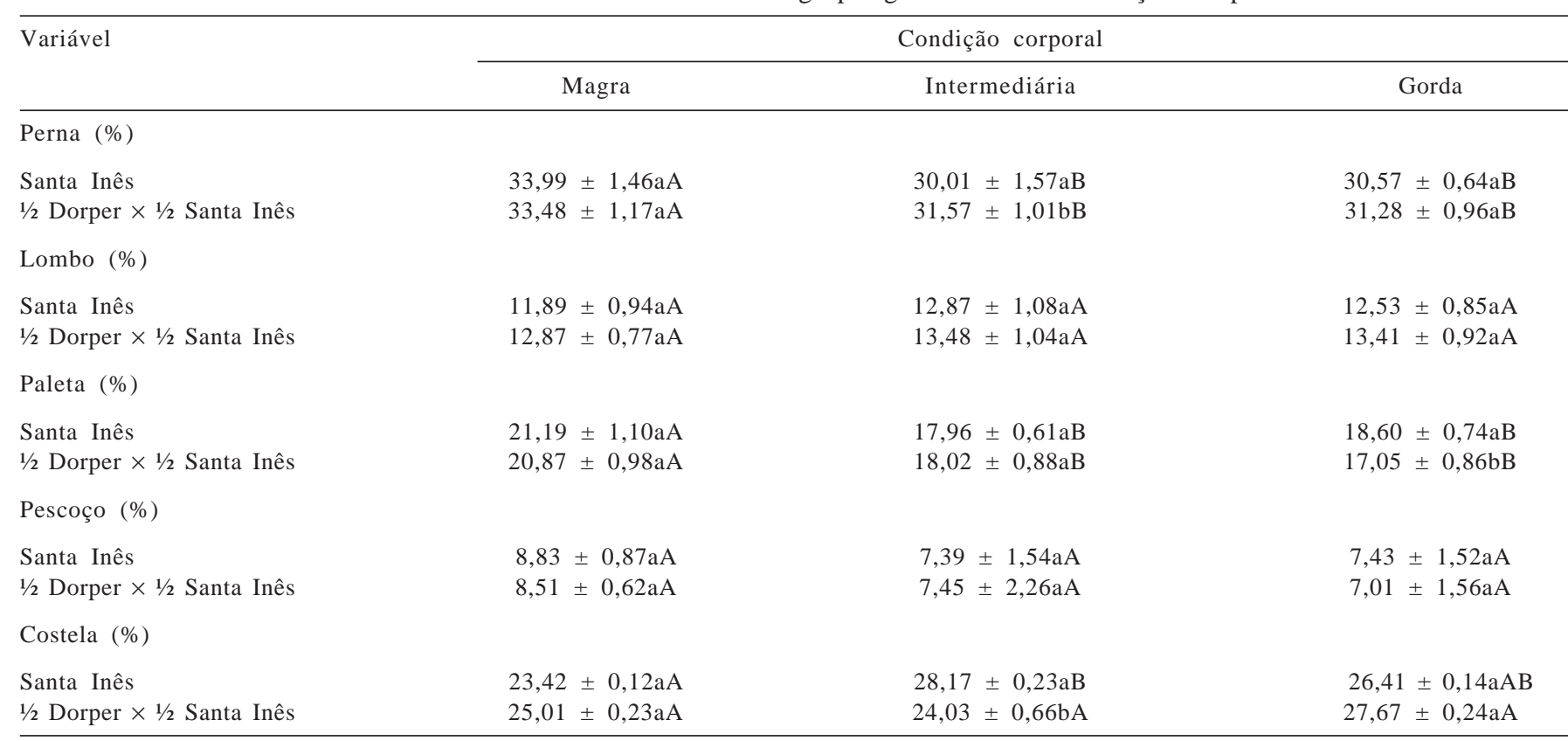

Letras distintas (minúscula na coluna e maiúscula na linha) indicam diferenças significativas pelo teste Tukey a 5\% de probabilidade.

Condição corporal: magra (escore corporal 1,0 a 2,0); intermediária (escore corporal 2,5 a 3,5); gorda (escore corporal 4,0 a 5,0).

\section{Conclusões}

O aumento da condição corporal melhorou o peso vivo ao abate, os pesos de carcaça quente e fria e os rendimentos de carcaça, independentemente do grupo genético. Cordeiros Santa Inês são mais precoces quanto à deposição de gordura interna que os cordeiros F1 Dorper $\times$ Santa Inês. Na condição corporal gorda, cordeiros F1 Dorper $\times$ Santa Inês apresentam, em relação aos Santa Inês, maior espessura de gordura subcutânea e carcaças mais bem acabadas. Na condição corporal intermediária, os cordeiros F1 Dorper $\times$ Santa Inês apresentam maior rendimento de perna que os Santa Inês.

\section{Literatura Citada}

ALMEIDA JR., G.A.; COSTA, C.; MONTEIRO, A.L.G. et al. Qualidade da carne de cordeiros criados em creep feeding com silagem de grãos úmidos de milho. Revista Brasileira de Zootecnia, v.33, n.4, p.1039-1047, 2004.

BONAGURIO, S.; PÉREZ, J.R.O.; GARCIA, I.F.F. et al. Qualidade da carne de cordeiros Santa Inês puros e mestiços com Texel, abatidos com diferentes pesos. Revista Brasileira de Zootecnia, v.32, n.6, p.1981-1991, 2003.

BUENO, S.M.; CUNHA, E.A.; SANTOS, L.E. et al. Características de carcaça de cordeiros Suffolk abatidos em diferentes idades. Revista Brasileira de Zootecnia, v.29, n.6, p.1803-1810, 2000.

BURKE, J.M.; APPLE, J.K. Growth performance and carcass traits of forage-fed hair sheep wethers. Small Ruminant Research, v.67, 264-270, 2007. 
FRESCURA, R.B.M.; PIRES, C.C.; SILVA, J.H.S. et al. Avaliação das proporções dos cortes da carcaça, características da carne e avaliação dos componentes do peso vivo de cordeiros. Revista Brasileira de Zootecnia, v.34, n.1, p.167-174, 2005.

FRUTOS, P.; MANTECON, A.R.; REVESADO, P.R. et al. Body fat depots and body condicion relations comparison of two spanish sheep breeds (Churra vs Merina). Options Méditerranéennes, p.19-23, 1995.

FURUSHO-GARCIA, I.F.F.; PEREZ, J.R.O.; OLIVEIRA, M.V. Características de carcaça de cordeiros Texel x Bergamácia, Texel x Santa Inês e Santa Inês Puros, terminados em confinamento, com casa de café como parte da dieta. Revista Brasileira de Zootecnia, v.29, n.1, p.253-260, 2000.

GUTIÉRREZ, J.; RUBIO, M.S.; MÉNDEZ, R.D. Effects of crossbreeding Mexican Pelibuey sheep with Rambouillet and Suffolk on carcass traits. Meat Science, v.70 p.1-5, 2005.

OLIVEIRA, M.V.M.; PÉREZ, J.R.O.; ALVES, E.L. et al. Rendimento de carcaça, mensurações e peso de cortes comerciais de cordeiros Santa Inês e Bergamácia alimentados com dejetos de suínos em confinamento. Revista Brasileira de Zootecnia, v.31, n.3, p.1451-1458, 2002 (supl.).

OSÓRIO, J.C.S.; OSÓRIO, M.T.M. Produção de carne ovina: técnicas de avaliação “in vivo” e na carcaça. Pelotas, 2003. 73p.

OSÓRIO, J.C.S.; OLIVEIRA, N.M.; OSÓRIO, M.T.M. et al. Produção de carne em cordeiros cruza Border Leicester com ovelhas Corriedale e Ideal. Revista Brasileira de Zootecnia, v.31, n.3, p.1469-1480, 2002 (supl.).

NATIONAL RESEARCH COUNCIL - NRC. Nutrient requirements of sheep. 6.ed. Washington, D.C.: National Academy Press, 1985. 99p.

NOTTER, D.R.; GREINER, S.P.; WAHLBERG, M.L. Growth and carcass characteristics of lamb sired by Dorper and Dorset rams. Journal of Animal Science, v.82, p.1323-1328, 2004.

PEÑA, F.; CANOB, T.; DOMENECHA, V. et al. Influence of sex, slaughter weight and carcass weight on” non-carcass” and carcass quality in Segureña lambs. Small Ruminant Research, v.60, p.247-254, 2005.

PURCHAS, R.W.; DAVIES, A.S.; ABDUKKAH, A.Y. An objective measure of muscularity: changes with animal growth and differences between genetic lives of southdown sheep. Meat Science, v.30, p.81-94, 1991.

REIS, W.; JOBIM, C.C.; MACEDO, F.A.F. et al. Características da carcaças de cordeiros alimentados com dietas contendo grãos de milho conservados em diferentes formas. Revista Brasileira de Zootecnia, v.30, n.4, p.1308-1315, 2001.

SIQUEIRA, E.R.; FERNANDES, S. Efeito do grupo genético sobre as medidas objetivas e subjetivas da carcaça de cordeiros terminados em confinamento. Revista Brasileira de Zootecnia, v.29, n.1, p.306-311, 2000.

SILVA SOBRINHO, A.G.; PURCHAS, R.W.; KADIM, I.T. et al. Musculosidade e composição da perna de ovinos de diferentes grupos genéticos e idades ao abate. Pesquisa Agropecuária Brasileira, v.40, n.11, p.1129-1134, 2005.

SNOWDER, G.D.; DUCKETT, S.K. Evaluation of the South African Dorper as a terminal sire breed for grown, carcass, and palatability characteristics. Journal of Animal Science, v.81, p.368-375, 2003.

SOUSA W.H.; LÔBO, R.N.B.; MORAIS, O.R. Ovinos Santa Inês: estado de arte e perspectivas. In: SIMPÓSIO INTERNACIONAL SOBRE CAPRINOS E OVINOS DE CORTE, 2003, João Pessoa. Anais... João Pessoa: SINCORTE, 2003. p.501-522.

SOUSA, W.H.; LEITE, P.R.M. Ovinos de corte: a raça Dorper. João Pessoa: EMEPA, 2000. 76p. 